FACTA UNIVERSITATIS (NIŠ)

Ser. Math. Inform. Vol. 35, No 5 (2020), 1399-1416

https://doi.org/10.22190/FUMI2005399S

\title{
A HYBRID ALGORITHM FOR THE UNCERTAIN INVERSE $p$-MEDIAN LOCATION PROBLEM
}

\author{
Akram Soltanpour, Fahimeh Baroughi and Behrooz Alizadeh
}

(C) 2020 by University of Niš, Serbia | Creative Commons Licence: CC BY-NC-ND

\begin{abstract}
In this paper, we investigate the inverse $p$-median location problem with variable edge lengths and variable vertex weights on networks in which the vertex weights and modification costs are the independent uncertain variables. We propose a model for the uncertain inverse $p$-median location problem with tail value at risk objective. Then, we show that it is NP-hard. Therefore, a hybrid particle swarm optimization algorithm has been presented to obtain the approximate optimal solution of the proposed model. The algorithm contains expected value simulation and tail value at risk simulation.

Keywords: p-median location problem; inverse optimization; Hybrid algorithm; nonlinear programming.
\end{abstract}

\section{Introduction}

One of the important aspects of location problems which has recently been studied by many researchers is the $p$-median location problem which can be stated as follows. Let $N=(V, E)$ be an undirected connected network with vertex set $V,|V|=n$, and edge set $E,|E|=m$. The distance between two points on $N$ is equal to the length of the shortest path connecting these two points. Each vertex is associated with a nonnegative weight that is the demand of the client at this vertex. In a $p$-median problem on a network, the aim is to find $p$ locations for establishing facilities on edges or vertices of the network such that the sum of the weighted distances from the clients to the closest facility becomes minimum. In the context of the $p$-median location problems, the interested reader is referred to papers $[1,7,8,11,12,13,17,20,21,28,37,39]$.

In recent years, inverse location problems have found an increasing interest. In an inverse location problem the goal is to modify parameters of the problem at

Received October 20, 2019; accepted November 13, 2020

2020 Mathematics Subject Classification. Primary 90C27; Secondary 90B80, 90C70, 90C59 
minimum cost such that a prespecified solution becomes optimal. Burkard et al. investigated the inverse 1-median problem with variable vertex weights on a tree network and also on a plane and presented algorithms in $O(n \log n)$ time for them [9]. Also they proposed an algorithm in $O\left(n^{2}\right)$ time for the problem under investigation on cycles [10]. Baroughi et al. [3] proved that the inverse $p$-median location problem (I $p$ MLP) on general networks is NP-hard. For a survey on the inverse $p$-median location problems, we refer the interested reader to $[16,18,19,23,30,36]$.

In the real life, we are usually faced with various types of uncertainty. For example, in location problems, we are usually not sure of the vertex weights, the travel times between vertices, the establishing costs of facilities and the vertex weights or edge lengths modification costs of a network. The uncertainty theory that proposed by Liu [25] is a suitable tool to deal with these parameters. Some researchers applied the uncertainty theory to deal with the location problems,for example Gao [14] modeled the single facility location problems with uncertain demands. Wen et al. [43] investigated the capacitated facility location-allocation problem with uncertain demands and also Nguyen and Chi [31] studied inverse 1-median problem on a tree with uncertain costs and showed that the inverse distribution function of the minimum cost can be obtained at $O\left(n^{2} \log n\right)$ time. For a survey on uncertain location problems, we refer the interested reader to $[15,22,27,34,40,46]$.

The uncertainty leads to the risk. Liu in [26] introduced the risk concept in the uncertain environment. Measuring the risk is one of the important steps in the decision making process. The risk metrics contain techniques and data sets used to calculate the risk value of the problem under investigation. Tail value at risk (TVaR) metric [32] is one of the measures of the risk that is widely acceptable among industry segments and market participants.

In the risk management related to location problems, Berman et al. [6] studied the effect of a decision maker's risk attitude on the median and center location problems, with uncertain demand in the mean-variance framework. Wang et al. [41] investigated a two-stage fuzzy facility location problem with value at risk. Wagner et al. [42] developed and examined a new algorithm for solving the p-median problem when the demands are probabilistic and correlated. For a survey on the risk management in the location problems with fuzzy variables, see, e.g. [5, 44].

In this paper, we concentrate on I $p$ MLP with variable edge lengths and variable vertex weights on networks. We assume that the vertex weights and modification costs are the independent uncertain variables. We propose a model for the uncertain inverse $p$-median location problem with tail value at risk objective and expected value constraints and show that the problem is NP-hard. Considering the uncertain and NP-hard nature in uncertain I $p$ MLP (UI $p$ MLP), evolutionary and meta-heuristics algorithms can be used to UI $p$ MLP for successful generation of optimal solutions. Hence, we present a hybrid particle swarm optimization algorithm which contains expected value simulation and tail value at risk simulation to obtain the approximate optimal solution of the proposed model.

Based on our knowledge, there are two papers on the implementation of metaheuristic algorithms to the inverse location problems until now. Alizadeh and 
Bakhteh [2] studied the general IpMLPs on networks and presented a modified firefly algorithm for the problem under investigation. Mirzapolis Adeh et al. [29] investigated the general inverse ordered $p$-median location problem on crisp networks and designed a modified particle swarm optimization (PSO) algorithm for it. There is no scientific paper on implementation of hybrid metaheuristic algorithms on I $p$ MLPs in uncertain networks. However, many papers can be found in the literature for other classical location problems on uncertain networks. Bashiri et al. [4] modeled fuzzy capacitated $p$-hub center problem and presented a genetic algorithm for the problem. Huang and Hao [22] modeled uncapacitated facility location problem with uncertain customers positions and provided a hybrid intelligent algorithm for solving it. In 2018 Rahmaniani et al. [35] proposed an efficient hybrid solution algorithm for the capacitated facility location-allocation problem under uncertainty. Yang et al. [45] presented an improved hybrid particle swarm optimization algorithm for fuzzy $p$-hub center problem.

The article is organized as follows: In the next section, we first introduce uncertainty theory and TVaR metric in an uncertain environment. Then, we discuss uncertain optimization model and present a new model with TVaR objective and expected value constraints. In Section 3., we first introduce I $p$ MLP with variable edge lengths and variable vertex weights on networks and then investigate the problem with uncertain vertex weights and uncertain modification costs. A model for the uncertain inverse $p$-median location problem (UI $p$ MLP) with TVaR objective is presented and it is shown that the problem under investigation is NP-hard. Then, we present a hybrid PSO algorithm to obtain the approximate optimal solution of the proposed model, which it contains expected value simulation and TVaR simulation. Finally, to show the effectiveness of the proposed hybrid PSO algorithm, we give a numerical example. Section 4 . gives a brief conclusion to this paper.

\section{Preliminaries}

In this section, we first present some definitions and theorems of the uncertainty theory and TVaR metric in an uncertain environment. Then, we introduce the uncertain optimization model and present a new model with TVaR objective and expected value constraints.

\subsection{Uncertainty theory}

Let $\Gamma$ be a nonempty set and $\Theta$ be a $\sigma$-algebra over $\Gamma$. An uncertain measure is a set function $\mathcal{M}: \Theta \rightarrow[0,1]$ that satisfies in normality, duality and subadditivity axioms. The triple $(\Gamma, \Theta, \mathcal{M})$ is called an uncertainty space.

Definition 2.1. ( $\operatorname{Liu}[25])$. Let $(\Gamma, \Theta, \mathcal{M})$ be an uncertainty space. A measurable function $\theta$ from $(\Gamma, \Theta, \mathcal{M})$ to the set of real numbers is called an uncertain variable. 
Definition 2.2. $(\operatorname{Liu}[25])$. Let $\theta$ be an uncertain variable. For any real number $x$, the function $\Upsilon(x)=\mathcal{M}\{\theta \leq x\}$ is called an uncertainty distribution of $\theta$.

Definition 2.3. $(\operatorname{Liu}[25])$. Let $\theta_{i}, i=1, \ldots, n$, be the uncertain variables. We call $\theta_{i}, i=1, \ldots, n$, independent if for any Borel sets $B_{1}, B_{2}, \ldots, B_{n}$ of real numbers,

$$
\mathcal{M}\left\{\bigcap_{i=1}^{n}\left\{\theta_{i} \in B_{i}\right\}\right\}=\bigwedge_{i=1}^{n} \mathcal{M}\left\{\theta_{i} \in B_{i}\right\} .
$$

Definition 2.4. $(\operatorname{Liu}[25])$. The expected value of the uncertain variable $\theta$ is defined as

$$
E[\theta]=\int_{0}^{+\infty} \mathcal{M}\{\theta \geq r\} d r-\int_{-\infty}^{0} \mathcal{M}\{\theta \leq r\} d r,
$$

provided that at least one of the two integral is finite.

A real valued function $f\left(x_{1}, x_{2}, \ldots, x_{n}\right)$ is said to be strictly increasing if $f\left(x_{1}, x_{2}, \ldots, x_{n}\right)>f\left(y_{1}, y_{2}, \ldots, y_{n}\right)$ when $x_{i}>y_{i}$ for $i=1,2, \ldots, n$.

Theorem 2.1. (Liu[25]). Let $\theta_{i}, i=1,2, \ldots, n$, be the independent uncertain variables and $\Upsilon_{i}^{-1}, i=1,2, \ldots, n$, be the inverse uncertainty distributions of $\theta_{i}$. Also, let $f\left(x_{1}, x_{2}, \ldots, x_{n}\right)$ be a strictly increasing function with respect to $x_{i}, i=$ $1,2, \ldots, n$. Then the uncertain variable $\vartheta=f\left(\theta_{1}, \theta_{2}, \ldots, \theta_{n}\right)$ has the following inverse uncertainty distribution

$$
\Upsilon^{-1}(\alpha)=f\left(\Upsilon_{1}^{-1}(\alpha), \ldots, \Upsilon_{n}^{-1}(\alpha)\right),
$$

and also it has the following expected value

$$
E[\vartheta]=\int_{0}^{1} f\left(\Upsilon_{1}^{-1}(\alpha), \ldots, \Upsilon_{n}^{-1}(\alpha)\right) d \alpha
$$

\subsection{TVaR metric in an uncertain environment}

The risk demonstrates a situation, in which there is a chance of loss or danger. The quantification of the risk is a key step towards the management and mitigation of the risk. In this section, we introduce the definition of the TVaR metric to account the probability of loss and the severity of the loss in an uncertain environment [32].

In order to define the TVaR metric, we first introduce the definition of the loss function.

Definition 2.5. $(\operatorname{Liu}[26])$. Consider $\theta_{i}, i=1,2, \ldots, n$, as the uncertain factors of a system. A function $f$ is said to be a loss function if some specified loss occurs if and only if

$$
f\left(\theta_{1}, \theta_{2}, \ldots, \theta_{n}\right)>0 .
$$


In the uncertain environment, TVaR of the loss function is defined as follows.

Definition 2.6. (Peng[32]). Let $\theta_{i}, i=1,2, \ldots, n$, be the uncertain factors and $f$ be the loss function of a system. Then TVaR of $f$ is defined as

$$
T V a R_{\beta}=\frac{1}{\beta} \int_{0}^{\beta} \sup \left\{\lambda \mid \mathcal{M}\left\{f\left(\theta_{1}, \theta_{2}, \ldots, \theta_{n}\right) \geq \lambda\right\} \geq \gamma\right\} d \gamma
$$

for each given risk confidence level $\beta \in(0,1]$.

Theorem 2.2. (Peng[32]). Let $\theta_{i}, i=1,2, \ldots, n$, be the uncertain factors of a system and $\Upsilon_{i}^{-1}, i=1,2, \ldots, n$, be the inverse uncertainty distributions of $\theta_{i}$. Also assume that the loss function $f\left(x_{1}, x_{2}, \ldots, x_{n}\right)$ is a strictly increasing function with respect to $x_{i}, i=1,2, \ldots, n$. Then, for each risk confidence level $\beta \in(0,1]$, we have

$$
T V a R_{\beta}=\frac{1}{\beta} \int_{0}^{\beta} f\left(\Upsilon_{1}^{-1}(1-\gamma), \Upsilon_{2}^{-1}(1-\gamma), \ldots, \Upsilon_{n}^{-1}(1-\gamma)\right) d \gamma
$$

\subsection{Uncertainty optimization}

Let $x=\left(x_{1}, x_{2}, \ldots, x_{n}\right)$ be a decision vector, and $\theta=\left(\theta_{1}, \theta_{2}, \ldots, \theta_{n}\right)$ be an uncertain vector. Consider the following optimization model.

$$
\begin{array}{cll}
\min & f(x, \theta) & \\
\mathrm{s.t.} & g_{j}(x, \theta) \leq 0 & j=1, \ldots, p, \\
& z_{l}(x) \leq 0 & l=1, \ldots, m, \\
& x \geq 0, &
\end{array}
$$

where $f$ and $g_{j}, j=1, \ldots, p$ are uncertain functions and $z_{l}, l=1, \ldots, m$ are crisp functions.

Since the objective function of the model (2.1) involves uncertainty, it cannot be directly optimized. Therefore, by considering $f(x, \theta)$ as a loss function, we minimize its TVaR. In addition, since the uncertain constraints do not define a crisp feasible set, we use the expected value of constraints. Thus, the model (2.1) can be reformulated as

$$
\begin{array}{cll}
\min & T V a R_{\alpha}(f(x, \theta)) & \\
\mathrm{s.t.} & E\left(g_{j}(x, \theta)\right) \leq 0 & j=1, \ldots, p, \\
& z_{l}(x) \leq 0 & l=1, \ldots, m, \\
& x \geq 0 . &
\end{array}
$$

According to Theorems 2.1 and 2.2, we can rewrite the problem (2.2) as follows:

$$
\begin{aligned}
\min & \frac{1}{\beta} \int_{0}^{\beta} f\left(x, \Upsilon_{1}^{-1}(1-\gamma), \Upsilon_{2}^{-1}(1-\gamma), \ldots, \Upsilon_{n}^{-1}(1-\gamma)\right) d \gamma \\
\text { s.t. } & \int_{0}^{1} g_{j}\left(x, \Upsilon_{1}^{-1}(\alpha), \Upsilon_{2}^{-1}(\alpha), \ldots, \Upsilon_{n}^{-1}(\alpha)\right) d \alpha \leq 0 \quad j=1, \ldots, p, \\
& z_{l}(x) \leq 0 \quad l=1, \ldots, m, \\
& x \geq 0,
\end{aligned}
$$

where $g_{j}\left(x, \theta_{1}, \theta_{2}, \ldots, \theta_{n}\right)$ is strictly increasing with respect to $\theta_{1}, \theta_{2}, \ldots, \theta_{n}$. 


\section{Problem definition}

In this section, we first introduce I $p$ MLP with variable edge lengths and variable vertex weights on networks and then investigate the problem with uncertain vertex weights and uncertain modification costs. A model for UI $p$ MLP with TVaR objective and expected value constraints is presented. To solve the proposed model, we present a hybrid PSO algorithm which contains expected value simulation and TVaR simulation.

\subsection{UI $p$ MLP on networks}

We can express I $p$ MLP with variable edge lengths and variable vertex weights as follows: Let $N=(V, E)$ with $|V|=n$ and $|E|=m$ be a connected network. Also let vertex $v \in V$ have a positive weight $w(v)$ and edge $e \in E$ have a positive length $\ell_{e}$. In an I $p$ MLP on networks, a set of vertices $\left\{m_{1}, \ldots, m_{p}\right\}$ is given. The goal is to modify $w(v), v \in V$, and $\ell_{e}, e \in E$, at minimum total cost such that the given set becomes a $p$-median of modified location problem. Let us consider nonnegative $\operatorname{costs} c_{e}^{+}$and $c_{v}^{+}$, if $\ell_{e}$ and $w(v)$ are increased by one unit, respectively. Also we consider nonnegative $\operatorname{costs} c_{e}^{-}$and $c_{v}^{-}$, if $\ell_{e}$ and $w(v)$ are decreased by one unit, respectively. Let $p_{e}, q_{e}, p_{v}$ and $q_{v}$ be the amounts by which the edge length $\ell_{e}$ and the vertex weight $w(v)$ are increased and decreased, respectively. We let $p_{e}, q_{e}, p_{v}$ and $q_{v}$ obey the upper bounds $u_{e}^{+}, u_{e}^{-}, u_{v}^{+}, u_{v}^{-}$. In addition, assume that $\mathcal{S}$ is the set of all subsets $S \subseteq V$ with $|S|=p$. Thus, I $p$ MLP on $N$ can be stated as follows.

Change $\ell_{e}, e \in E$, to $\tilde{\ell}_{e}=\ell_{e}+p_{e}-q_{e}$ and $w(v), v \in V$, to $\tilde{w}(v)=w(v)+p_{v}-q_{v}$ such that

(i) The set $\left\{m_{1}, \ldots, m_{p}\right\}$ becomes a $p$-median of $N$ with respect to $\tilde{\ell}$ and $\tilde{w}(v)$, i.e.,

$$
\sum_{v \in V} \tilde{w}(v) \min _{i=1, \ldots, p} d_{\tilde{\ell}}\left(v, m_{i}\right) \leq \sum_{v \in V} \tilde{w}(v) \min _{k \in S} d_{\tilde{\ell}}\left(v, v_{k}\right) \quad \forall S \in \mathcal{S}
$$

(ii) The bound constraints are satisfied:

$$
\begin{aligned}
& 0 \leq p_{e} \leq u_{e}^{+}, \quad 0 \leq q_{e} \leq u_{e}^{-} \quad \forall e \in E, \\
& 0 \leq p_{v} \leq u_{v}^{+}, \quad 0 \leq q_{v} \leq u_{v}^{-} \quad \forall v \in V,
\end{aligned}
$$

(iii) The objective function

$$
\sum_{e \in E}\left(c_{e}^{+} p_{e}+c_{e}^{-} q_{e}\right)+\sum_{v \in V}\left(c_{v}^{+} p_{v}+c_{v}^{-} q_{v}\right)
$$

becomes minimum. 
This formulation of I $p$ MLP is a nonlinear programming model. In the following, we consider I $p$ MLP with uncertain vertex weights and uncertain modification costs.

Let $N=(V, E)$ be a network with independent uncertain vertex weights $\theta_{v}, v \in V$. Also let $w(v)$ be a parameter on each vertex $v \in V$, which will be changed to $\tilde{w}(v)$. In addition, suppose that $\theta_{v}$ relates to this parameter, i.e., for each vertex $v \in V$, we have an original weight $\theta(w(v))$ and also a new weight $\theta(\tilde{w}(v))$. Let $\vartheta_{v}^{+}$and $\vartheta_{v}^{-}$ be the independent uncertain variables with respect to the $\operatorname{costs} c_{v}^{+}$and $c_{v}^{-}$, for all $v \in V$, and $\vartheta_{e}^{+}$and $\vartheta_{e}^{-}$be the independent uncertain variables with respect to the $\operatorname{costs} c_{e}^{+}$and $c_{e}^{-}$, for all $e \in E$, respectively.

Let us assume that we are given a set of vertices $\left\{m_{1}, \ldots, m_{p}\right\}$. In an UI $p$ MLP, the goal is to find $\tilde{\ell}_{e}=\ell_{e}+p_{e}-q_{e}$ and $\tilde{w}(v)=w(v)+p_{v}-q_{v}$ such that $\left\{m_{1}, \ldots, m_{p}\right\}$ becomes a $p$-median of the problem with respect to $\theta_{v}(\tilde{w}(v))$ and $\tilde{\ell}_{e}, v \in V, e \in E$, and the total cost

$$
\sum_{v \in V}\left(\vartheta_{v}^{+} p_{v}+\vartheta_{v}^{-} q_{v}\right)+\sum_{e \in E}\left(\vartheta_{e}^{+} p_{e}+\vartheta_{e}^{-} q_{e}\right)
$$

is minimized.

Therefore, we can model UI $p$ MLP as follows.

$$
\begin{array}{cl}
\min & {\left[\sum_{v \in V}\left(\vartheta_{v}^{+} p_{v}+\vartheta_{v}^{-} q_{v}\right)+\sum_{e \in E}\left(\vartheta_{e}^{+} p_{e}+\vartheta_{e}^{-} q_{e}\right)\right]} \\
\text { s.t. } & \\
(3.4) \quad\left[\sum_{v \in V} \theta(\tilde{w}(v))\left(\min _{i=1, \ldots, p} d_{\tilde{\ell}}\left(v, m_{i}\right)-\min _{k \in S} d_{\tilde{\ell}}\left(v, v_{k}\right)\right)\right] \leq 0 \quad \forall S \in \mathcal{S}, \\
& 0 \leq p_{e} \leq u_{e}^{+}, \quad 0 \leq q_{e} \leq u_{e}^{-} \quad \forall e \in E, \\
& 0 \leq p_{v} \leq u_{v}^{+}, \quad 0 \leq q_{v} \leq u_{v}^{-} \quad \forall v \in V .
\end{array}
$$

Definition 3.1. Let $p=\left(p_{e}\right)_{e \in E}$ and $q=\left(q_{v}\right)_{v \in V}$ be the vectors that satisfies in (3.2) and (3.3). Then $(p, q)$ is called expected solution of (3.4) if and only if $\forall S \in \mathcal{S}$

$$
\sum_{v \in V} E[\theta(\tilde{w}(v))]\left(\min _{i=1, \ldots, p} d_{\tilde{\ell}}\left(v, m_{i}\right)-\min _{k \in S} d_{\tilde{\ell}}\left(v, v_{k}\right)\right) \leq 0 .
$$

Now, let $(p, q)$ be a expected solution of (3.4). Define

$$
f(p, q)=\sum_{v \in V}\left(\vartheta_{v}^{+} p_{v}+\vartheta_{v}^{-} q_{v}\right)+\sum_{e \in E}\left(\vartheta_{e}^{+} p_{e}+\vartheta_{e}^{-} q_{e}\right) .
$$

Definition 3.2. For a risk confidence level $\beta \in(0,1]$, a expected solution $\left(p^{*}, q^{*}\right)$ is called optimal solution with minimum $T V a R$ if

$$
T \operatorname{VaR} R_{\beta}\left(f\left(p^{*}, q^{*}\right)\right) \leq T \operatorname{VaR} \beta(f(p, q)),
$$

holds for any expected solution $(p, q)$.

Therefore, we can find an optimal expected solution with minimum TVaR as follows: 
Let $\left(\Psi_{v}^{+}\right)^{-1}, v \in V$, and $\left(\Psi_{e}^{+}\right)^{-1}, e \in E$ be the inverse uncertainty distributions of $\vartheta_{v}^{+}$and $\vartheta_{e}^{+}$, respectively. Also let $\left(\Psi_{v}^{-}\right)^{-1}, v \in V$, and $\left(\Psi_{e}^{-}\right)^{-1}, e \in E$ be the inverse uncertainty distributions of $\vartheta_{v}^{-}$and $\vartheta_{e}^{-}$, respectively. Assume that $\Upsilon_{v}^{-1}$, $v \in V$, is the inverse uncertainty distribution of $\theta_{v}$. Then, for a risk confidence level $\beta \in(0,1]$, the optimal expected solution with minimum TVaR is the optimal solution of the following model:

$$
\begin{array}{ll}
\min & \sum_{v \in V}\left[\left(\frac{1}{\beta} \int_{0}^{\beta}\left(\Psi_{v}^{+}\right)^{-1}(1-\gamma) d \gamma\right) p_{v}+\left(\frac{1}{\beta} \int_{0}^{\beta}\left(\Psi_{v}^{-}\right)^{-1}(1-\gamma) d \gamma\right) q_{v}\right] \\
& +\sum_{e \in E}\left[\left(\frac{1}{\beta} \int_{0}^{\beta}\left(\Psi_{e}^{+}\right)^{-1}(1-\gamma) d \gamma\right) p_{e}+\left(\frac{1}{\beta} \int_{0}^{\beta}\left(\Psi_{e}^{-}\right)^{-1}(1-\gamma) d \gamma\right) q_{e}\right] \\
\text { s.t. } & \\
& \sum_{v \in V}\left(\int_{0}^{1} \Upsilon_{v}^{-1}(\tilde{w}(v), \alpha) d \alpha\right)\left(\min _{i=1, \ldots, p} d_{\tilde{\ell}}\left(v, m_{i}\right)-\min _{k \in S} d_{\tilde{\ell}}\left(v, v_{k}\right)\right) \leq 0 \\
& 0 \leq p_{e} \leq u_{e}^{+}, \quad 0 \leq q_{e} \leq u_{e}^{-} \quad \forall e \in E, \\
& 0 \leq p_{v} \leq u_{v}^{+}, \quad 0 \leq q_{v} \leq u_{v}^{-} \quad \forall v \in V,
\end{array}
$$

The above model is a deterministic inverse $p$-median problem formulation with vertex weights

$$
\int_{0}^{1} \Upsilon_{v}^{-1}(\tilde{w}(v), \alpha) d \alpha, \quad \forall v \in V,
$$

vertex weight modification costs

$$
\frac{1}{\beta} \int_{0}^{\beta}\left(\Psi_{v}^{+}\right)^{-1}(1-\gamma) d \gamma, \frac{1}{\beta} \int_{0}^{\beta}\left(\Psi_{v}^{-}\right)^{-1}(1-\gamma) d \gamma,
$$

and edge length modification costs

$$
\frac{1}{\beta} \int_{0}^{\beta}\left(\Psi_{v}^{+}\right)^{-1}(1-\gamma) d \gamma, \frac{1}{\beta} \int_{0}^{\beta}\left(\Psi_{v}^{-}\right)^{-1}(1-\gamma) d \gamma
$$

Baroughi et al. in [3] showed that I $p$ MLP on general networks is NP-hard. Thus we immediately conclude the following proposition.

Proposition 3.1. UIpMLP with TVaR criterion on general networks is NP-hard.

The above proposition implies that it is not possible to present exact polynomial time methods to solve UI $p$ MLP on general networks. Therefore, we propose an efficient hybrid PSO algorithm for approximating the optimal solution of UI $p$ MP on networks. 


\subsection{Hybrid PSO algorithm}

Kennedy and Eberhart in 1995 [24] developed the PSO algorithm as a natureinspired evolutionary computation algorithm. Consider the following model

$$
\begin{array}{cl}
\min & f(x) \\
\text { s.t. } & x \in X,
\end{array}
$$

where $X$ is the restricted region. In PSO algorithm, a potential solution is presented as a particle $x_{j} \in X$ and a direction $v_{j} \in \mathbb{R}$ in which the particle will move. A swarm of particles is defined as a set $\left\{x_{1}, x_{2}, \ldots, x_{N}\right\}$, in which $N$ is number of particles. Each particle $x_{j}$ retains a record of the position of its previous best performance in a vector called $P_{b e s t, j}$. The particle with best performance $\mathrm{r}$ in the population has been maintained in a vector $G_{b e s t}$. An iteration involves evaluating of each particle $x_{j}$, then randomly setting of $v_{j}$ in the direction of particle $x_{j}^{\prime}$ s best previous position $P_{b e s t, j}$ and the best previous position $G_{b e s t}$ of any particle in the population.

Since in UI $p$ MLP the aim is to modify the vertex weights and edge lengths with respect to modification bounds. Thus, we consider a particle of the problem as $x_{j}=\left(x_{1, j}, x_{2, j}, \ldots, x_{2 m+2 n, j}\right)$ where

$$
\begin{aligned}
& \left(x_{1, j}, x_{2, j}, \ldots, x_{m, j}\right)=\left(p_{e}\right)_{e \in E} \\
& \left(x_{m+1, j}, x_{m+2, j}, \ldots, x_{2 m, j}\right)=\left(q_{e}\right)_{e \in E} \\
& \left(x_{2 m+1, j}, x_{2 m+2, j}, \ldots, x_{2 m+n, j}\right)=\left(p_{v}\right)_{v \in V}, \\
& \left(x_{2 m+n+1, j}, x_{2 m+n+2, j}, \ldots, x_{2 m+2 n, j}\right)=\left(q_{v}\right)_{v \in V} .
\end{aligned}
$$

Therefore, $x_{j}$ represents the decision vector of UI $p$ MLP that used in PSO. In addition, according to the orthogonality condition

- if $q_{e}>p_{e}$, then $q_{e}=q_{e}-p_{e}, p_{e}=0$,

- if $q_{e}<p_{e}$, then $p_{e}=p_{e}-q_{e}, q_{e}=0$,

- if $q_{v}>p_{v}$, then $q_{v}=q_{v}-p_{v}, p_{v}=0$,

- if $q_{v}<p_{v}$, then $p_{v}=p_{v}-q_{v}, q_{v}=0$.

For checking the feasibility of particle $x_{j}$, we calculate the expected value of constraints by using the following uncertain simulation algorithm [33]. Let $S \in \mathcal{S}$.

Algorithm 1 (Expected value simulation)

1. Set $E=0$.

2. For $k=1, \ldots, 99$ do

compute

$$
E_{k}=0.01 \sum_{v \in V}\left(\Upsilon_{v}^{-1}(\tilde{w}(v), 0.0 k)\right)\left(\min _{i=1, \ldots, p} d_{\tilde{\ell}}\left(v, m_{i}\right)-\min _{k \in S} d_{\tilde{\ell}}\left(v, v_{k}\right)\right),
$$


and $E:=E+E_{k}$.

\section{Report $E$.}

Therefore, if the particle $x_{j}=\left(x_{1, j}, x_{2, j}, \ldots, x_{2 m+2 n, j}\right)$ is defined as (3.6) and for each $S \in \mathcal{S}, E \leq 0$, then $x_{j}$ is feasibile.

Based on Theorem 2.2, we present the following uncertain simulation procedure for computing TVaR of objective function for each feasible particle $x_{j}$ and given $\beta \in(0,1]$.

\section{Algorithm 2 (TVaR simulation)}

1. Set $T_{\beta}=0$.

2. For $j=1, \ldots, M$ do

compute

$$
\begin{aligned}
& T_{\beta}^{j}=\sum_{v \in V}\left[\left(\left(\Psi_{v}^{+}\right)^{-1}\left(1-\frac{j}{M} \beta\right)\right) p_{v}+\left(\left(\Psi_{v}^{-}\right)^{-1}\left(1-\frac{j}{M} \beta\right)\right) q_{v}\right] \\
& \quad+\sum_{e \in E}\left[\left(\left(\Psi_{e}^{+}\right)^{-1}\left(1-\frac{j}{M} \beta\right)\right) p_{e}+\left(\left(\Psi_{e}^{-}\right)^{-1}\left(1-\frac{j}{M} \beta\right)\right) q_{e}\right], \\
& \text { and } T_{\beta}=T_{\beta}+\frac{j}{M} \beta T_{\beta}^{j} .
\end{aligned}
$$

3. Compute $T V a R_{\beta}=\frac{1}{\beta} T_{\beta}$.

\section{Report $T V a R_{\beta}$.}

To solve the model (3.5) with hybrid PSO algorithm, we first randomly generate the particle $x_{j}$ by checking the feasibility of it using expected value simulation. Repeat this process $N$ times. We get $N$ initial feasible particles $x_{1}, x_{2}, \ldots, x_{N}$. Then, we assume that the fitness of each $x_{j}$ is the minus of TVaR, i.e.,

$$
\operatorname{Fit}\left(x_{j}\right)=-T V a R_{\beta}\left(x_{j}\right) .
$$

Thus, the particle with higher fitness has smaller objective value. The fitness of each particle is obtained by using TVaR simulation.

In the process of updating $(i+1)$ th iteration, we first denote $P_{b e s t, j}(i)$ for each particle $x_{j}$ and $G_{b e s t}(i)$, then we obtain the new directs and the positions of the particles by using the following two equations:

$$
v_{j}(i+1)=v_{j}(i)+C_{1} r_{1}\left[P_{b e s t, j}(i)-x_{j}(i)\right]+C_{2} r_{2}\left[G_{b e s t}(i)-x_{j}(i)\right],
$$




$$
x_{j}(i+1)=x_{j}(i)+v_{j}(i+1)
$$

where, $P_{b e s t, j}(i)=x_{j}(i)$ if

$$
\operatorname{Fit}\left(x_{j}(i)\right) \geq \operatorname{Fit}\left(x_{j}(i-1)\right)
$$

and $P_{b e s t, j}(i)=P_{b e s t, j}(i-1)$ otherwise, and $G_{b e s t}(i)=P_{b e s t, k}(i)$, with

$$
k=\operatorname{argmin}\left\{P_{\text {best }, j}(i): j=1, \ldots, N\right\} .
$$

In addition $r_{1}$ and $r_{2}$ are uniformly distributed random numbers in the interval $[0,1]$ and $C_{1}$ and $C_{2}$ are learning rates, to well adjust the convergence of the particles. The values of $C_{1}$ and $C_{2}$ are usually assumed to be 2 .

If the updated $x_{j}$ is feasible, then we consider it as a new particle of the next generation. Otherwise, as long as a feasible new particle is found, we re-update (3.7) and (3.8).

We obtain a new generation of particles by repeating the above process $N$ times.

If MaxIt indicate the number of generations of the PSO algorithm, then based on all the explanations above, we summarize the hybrid PSO algorithm for solving the model (3.5) as follows.

\section{Algorithm 3 (Hybrid PSO algorithm)}

1. Initialize the feasibile particles $x_{1}, \ldots, x_{N}$ (use expected value simulation).

2. Compute the fitness for all particles by using TVaR simulation, and evaluate each particle according to it.

3. Update all the particles by using equations (3.7) and (3.8).

4. As long as a new feasible population is found, re-update (3.7) and (3.8).

5. Repeat Steps 2 to 4 for MaxIt times.

6. Return $G_{\text {best }}$ as the optimal solution of the model (3.5), and

$$
T \operatorname{VaR} R_{\beta}\left(G_{\text {best }}\right)=-\operatorname{Fit}\left(G_{\text {best }}\right)
$$

as the corresponding optimal value.

\subsection{An illustrative example}

In this subsection, we give a numerical example to illustrate the hybrid PSO algorithm. The result of the numerical experiment is obtained on a $\mathrm{PC}$ with processor Intel(R) Core(TM) i3 CPU 2.27GHZ and 4GB of RAM under windows 7.

We apply the hybrid PSO algorithm for solving UIpMLP with TVaR criteria at a risk confidence level of $\beta=0.8$ on the given network $N$ in Figure 3.1. Let the 
cost coefficients be linear uncertain variables (see Table 3.3). Also let the vertex weights $\theta$ be the linear uncertain variables with respect to, $\tilde{w}(v)$, i.e.,

$$
\theta=\theta(\tilde{w}(v))=\mathcal{L}(\tilde{w}(v)-10, \tilde{w}(v)+10) .
$$

The input data of the network are given in Tables 3.1 and 3.3.

Note that if $\theta=\mathcal{L}(a, b)$ is the linear uncertain variable, then for a risk confidence level $\beta \in(0,1]$

$$
T V a R_{\beta}(\theta)=\frac{\beta}{2}(a-b)+b,
$$

and

$$
E[\theta]=\frac{(a+b)}{2} .
$$

In the following, we show the computational results of the hybrid PSO algorithm's performance on an example of UI2MLP on the given network.

Note that the goal is to change $w(v)$ and $\ell_{e}$ with respect to modification bounds so that $\left\{v_{2}, v_{3}\right\}$ becomes a 2 -median at minimum total cost under the new vertex weights and edge lengths.

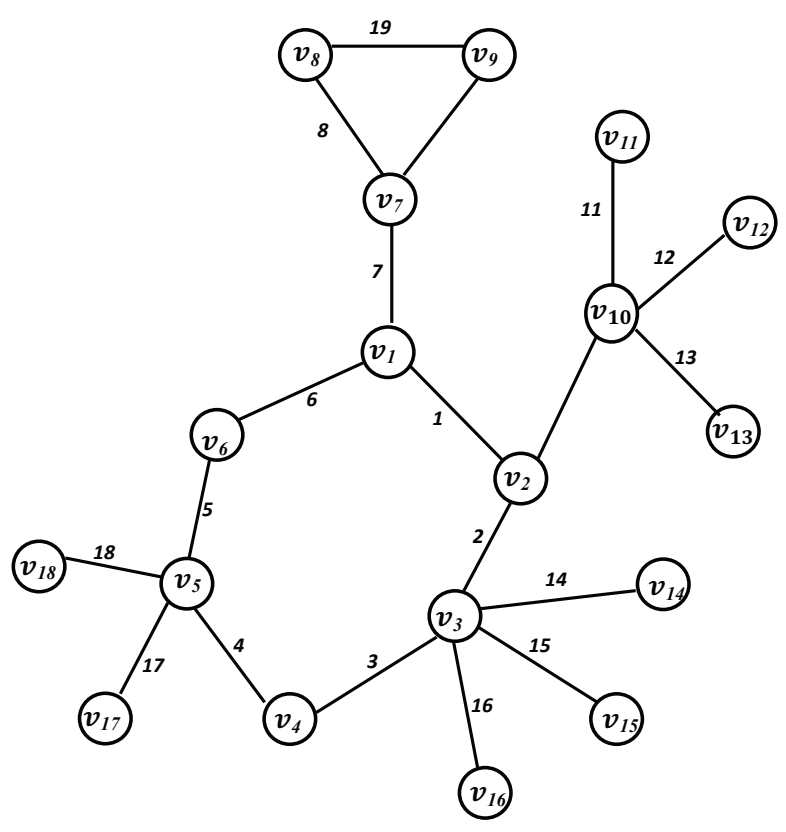

FIG. 3.1: Network $N$ 
Table 3.1: The input data for UI2MLP

\begin{tabular}{|c|c|}
\hline$\ell_{e}$ & $(14,34,25,7,22,10,8,20,12,7,10,26,12,6,10,23,31,21,22)$ \\
\hline$u_{e}^{+}$ & $(5,4,5,4,7,2,5,6,3,9,2,13,1,3,5,1,7,4,1)$ \\
\hline$u_{e}^{-}$ & $(10,30,15,3,17,8,4,10,8,4,5,20,6,1,8,13,2,3,13)$ \\
\hline$w(v)$ & $(34,18,14,13,21,11,13,20,40,22,9,17,13,6,24,14,15,12)$ \\
\hline$u_{v}^{+}$ & $(20,11,4,1,2,4,7,8,15,32,13,5,15,1,2,4,1,1)$ \\
\hline$u_{v}^{-}$ & $(3,2,11,9,10,8,1,1,6,7,2,2,2,4,15,10,13,11)$ \\
\hline
\end{tabular}

The hybrid PSO algorithm is run for the problem with 100, 200, 300 and 400 generations, respectively. Table 3.2 shows the best solutions of the problem.

Table 3.4, shows the best solutions of UI2MLP using hybrid PSO algorithm. Furthermore, the convergence of the objective values with population sizes 10,15,20,25 and MaxIt $=100$ is shown in Figure 3.2. The convergence of the objective values with $N=10$ and MaxIt $=100,200,300,400$ is given in Figure 3.3.

Table 3.2: The results of the performance of hybrid PSO algorithm

\begin{tabular}{|c|c|c|c|}
\hline N, MaxIt & Objective value & $\mathrm{N}$, MaxIt & Objective value \\
\hline \hline 10,100 & -6880 & 10,200 & -6635 \\
\hline 15,100 & -8240 & 10,300 & -7609 \\
\hline 20,100 & -8520 & 10,400 & -9150 \\
\hline 25,100 & -10496 & & \\
\hline
\end{tabular}

Table 3.3: Uncertain cost coefficients

\begin{tabular}{|c|c|}
\hline$\vartheta_{e}^{+}$ & $(\mathcal{L}(8,10), \mathcal{L}(18,21), \mathcal{L}(19,21), \mathcal{L}(4,6), \mathcal{L}(3,4), \mathcal{L}(14,16), \mathcal{L}(28,30), \mathcal{L}(10,12)$, \\
& $\mathcal{L}(17,18), \mathcal{L}(6,8), \mathcal{L}(22,24), \mathcal{L}(7,9), \mathcal{L}(15,17), \mathcal{L}(18,21), \mathcal{L}(26,28)$, \\
& $\mathcal{L}(28,30), \mathcal{L}(16,18), \mathcal{L}(4,6), \mathcal{L}(4,6))$ \\
\hline$\vartheta_{e}^{-}$ & $(\mathcal{L}(18,20), \mathcal{L}(14,15), \mathcal{L}(10,12), \mathcal{L}(24,26), \mathcal{L}(17,18), \mathcal{L}(15,17), \mathcal{L}(27,29), \mathcal{L}(8,10)$, \\
& $\mathcal{L}(22,24), \mathcal{L}(22,24), \mathcal{L}(11,13), \mathcal{L}(2,4), \mathcal{L}(1,3), \mathcal{L}(15,17), \mathcal{L}(24,25)$, \\
& $\mathcal{L}(28,30), \mathcal{L}(3,5), \mathcal{L}(17,19), \mathcal{L}(17,19))$ \\
\hline$\vartheta_{v}^{+}$ & $(\mathcal{L}(24,26), \mathcal{L}(27,28), \mathcal{L}(3,5), \mathcal{L}(27,28), \mathcal{L}(19,20), \mathcal{L}(1,4), \mathcal{L}(8,10), \mathcal{L}(16,18)$, \\
& $\mathcal{L}(29,30), \mathcal{L}(29,30), \mathcal{L}(4,6), \mathcal{L}(30,31), \mathcal{L}(29,30), \mathcal{L}(14,16), \mathcal{L}(24,26)$, \\
& $\mathcal{L}(4,6), \mathcal{L}(12,13), \mathcal{L}(27,28))$ \\
\hline$\vartheta_{v}^{-}$ & $(\mathcal{L}(19,21), \mathcal{L}(22,24), \mathcal{L}(12,13), \mathcal{L}(19,21), \mathcal{L}(5,6), \mathcal{L}(20,22), \mathcal{L}(1,2), \mathcal{L}(7,10)$, \\
& $\mathcal{L}(2,3), \mathcal{L}(3,4), \mathcal{L}(24,26), \mathcal{L}(20,21), \mathcal{L}(19,20), \mathcal{L}(29,30), \mathcal{L}(2,3)$, \\
& $\mathcal{L}(13,15), \mathcal{L}(12,13), \mathcal{L}(22,24))$ \\
\hline
\end{tabular}


Table 3.4: The obtained $G_{b e s t}$ for UI2MLP by using hybrid PSO algorithm

\begin{tabular}{|c|c|}
\hline $\mathrm{N}$, MaxIt & $G_{\text {best }}$ \\
\hline 10,100 & $\begin{array}{c}(3.92,0.90,4.88,0,4.84,0,0,5.82,1.25,0,0,0.33,0,0,0.99,0.94, \\
0.82,0.69,0,0,0,2.75,0,2.50,3.22,0,0,2.80,1.08,0,4.60,0.23,0, \\
0,1.46,0,0,0,9.51,3.40,0.32,1.38,0,0,0,0,0,0,4.94,0,0,1.54, \\
3.26,0.61,0,0.22,0,0,0,0,6.31,0.34,0.72,4.28,5.64,0.01,0,0.34,2.56, \\
0,0,0,10.73)\end{array}$ \\
\hline 15,100 & $\begin{array}{c}(0.24,2.85,4.07,0,4.07,1.29,1.95,4.12,0,8.92,0,8.38,0.97,0.30 \\
3.2,0,3.42,3.49,0.08,0,0,0,2.12,0,0,0,0,6.16,0,2.68,0,0,0,0 \\
7.84,0,0,0,0.50,0,1.95,0.30,0.94,3.70,6.81,0,0,7.51,5.21,1.95 \\
0,0,0,0,0,0,0,0.94,0,0,0,0,0,0.31,0.48,0,0,0,0.8,1.08,4.82 \\
8.40,5.80,10.03)\end{array}$ \\
\hline 20,100 & $\begin{array}{c}(3.43,3.91,1.20,0,4.02,0,0,4,2.57,0,1.48,11.32,0,0,3.03,0,0,0, \\
0,0,0,0,1.67,0,3.10,1.55,0,0,3.70,0,0,0.86,0.96,0,7.83,1.09, \\
1.22,2.76,0.52,0,0.78,0,0.44,1.79,6.47,0,11.03,0,0,0,4.89,0.02,0 \\
0,0.29,0,0,0.11,0,4.76,0,0,0,0.10,0,5.28,1.77,0.68,0,0,4.57,5.31 \\
0,10)\end{array}$ \\
\hline 25,100 & $\begin{array}{c}(2.32,2.36,2.88,0,4.76,0,0.28,0,0.80,0,1.78,0,0.16,0,2.61 \\
0,1.70,0,0.91,0,0,0,2.04,0,1.45,0,9.33,0,0.50,0,8.00,0,0.39,0 \\
7.15,0,1.06,0,0,8.81,0.69,0,0,0.93,1.37,4.04,3.59,26.46,0,0.82,0 \\
0,0,0,0.45,0,0.62,0,0,4.63,4.86,0,0,0,0,0,1.35,0,0.12,0.72 \\
12.62,3.096,0,10.65)\end{array}$ \\
\hline 10,200 & 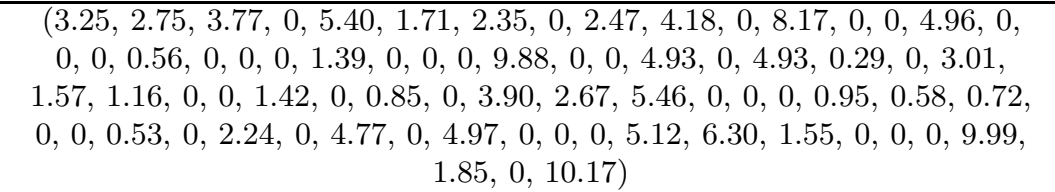 \\
\hline 10,300 & $\begin{array}{c}.42,0.91,2.20,0,2.82,0,0,0,2.54,0,0,1.42,0,0,0,0,4.70,0,0.77, \\
0,0,0,1.51,0,1.05,1.30,5.94,0,0.89,2.83,0,2.91,0.20,1.74,2.71 \\
0,2.60,0,0,3.66,0,0,0,0,6.34,6.11,9.43,0,0,1.10,0,0.20,1.64 \\
0.36,0,0,2.31,0,1.27,2.86,6.34,5.40,0,0,0,4.33,0.79,0,0.50,0 \\
0,0,0.81,10.66)\end{array}$ \\
\hline 10,400 & $\begin{array}{c}(1.80,2.94,1.64,0,3.60,0,2.13,4.58,0.53,0,0.36,11.20,0.42,1.08, \\
0,0,0,2.98,0,0,0,0,1.94,0,2.28,0,0,0,3.89,0,0,0,0,7.56,5.33, \\
1.80,0,9.03,1.69,8.02,1.17,0,0,3.97,1.14,7.81,0,1.61,4.24, \\
1.71,0,0.71,0.71,0,0.66,0,0,0,0,8.40,3.35,0,0,0,4.31,0,0,0, \\
1.43,0,0,4.72,0,10.97)\end{array}$ \\
\hline
\end{tabular}




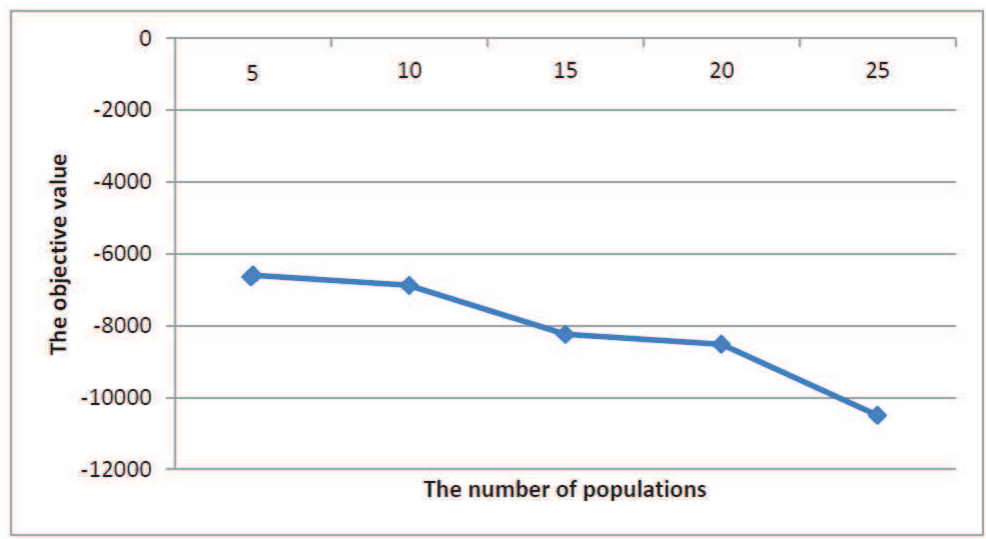

FIG. 3.2: The convergence of TVaR, MaxIt=100

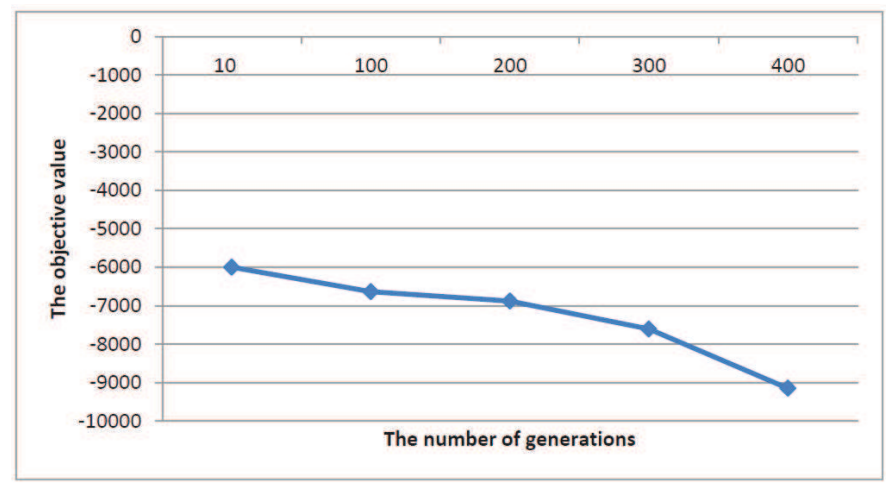

Fig. 3.3: The convergence of TVaR, $\mathrm{N}=10$

\section{Conclusion}

In this paper, we investigated IpMLP with variable edge lengths and variable vertex weights on a network in which the vertex weights and modification costs are the independent uncertain variables. We proposed a model for UI $p$ MLP with TVaR objective and expected value constraints and showed that it is NP-hard. Thus, we presented a hybrid PSO algorithm for approximating the optimal solutions, which it contains expected value simulation and TVaR simulation. Finally, by computational experiments, the efficiency of the algorithm is illustrated. 


\section{REFEREN C ES}

1. O. Alp, E. ERKut and Z. Drezner: An efficient genetic algorithm for the p-median problem. Ann. Oper. Res. 10 (2003), 1387-1395.

2. B. AlizAdeh and S. BAKHTEH: A modified firefly algorithm for general inverse p-median location problems under different distance norms. Opsearch. 54 (2017), 618636.

3. F. Baroughi, R. E. Burkard and E. Gassner: Inverse p-median problems with variable edge lengths. Math. Meth. Oper. Res. 73 (2011), 263-280.

4. M. Bashiri, M. Mirzaei and M. RAndall: Modeling fuzzy capacitated p-hub center problem and a genetic algorithm solution. Appl. Math. Model. 37(5) (2013), 3513-3525.

5. X. BAI and Y. LIU: Minimum risk facility location-allocation problem with type-2 fuzzy variables. sci. world j. 2014 (2014), 1-9.

6. O. Berman, N. SANAJIAN and J. WANG: Location choice and risk attitude of a decision maker. Omega 66 (2017), 170-181.

7. R. Benkoczi and B. Bhattacharya: A new template for solving p-median problems for trees in sub-quadratic time (extended abstract). LNCS. 3669 (2005), 271-282.

8. R. E. BURKARD and J. KRARUP: A linear algorithm for the pos/neg-weighted 1median problem on a cactus. Computing. 60 (1998), 193-215.

9. R. E. Burkard, C. Pleschiutschnig and J. Zhan: Inverse median problems. Discrete Optim. 1 (2004), 23-39.

10. R. E. Burkard, C. Pleschiutschnig and J. Zhan: The inverse 1-median problem on a cycle. Discrete Optim. 5 (2008), 242-253.

11. Z. DREZNER: The planar two-center and two-median problems. Transportation Sci. 18 (1984), 351-361.

12. S. Eilon, C. D. T. Watson-Gandy and N. Christofides: Distribution management: mathematical modeling and practical analysis. New York: Hafner (1971).

13. H. A. EISElT and V. MARIAnov: Foundations of location analysis. OR/MS, NewYork: Springer (2011).

14. Y. GAO : Uncertain models for single facility location problems on networks. Appl. Math. Model. 36 (2012), 2592-2599.

15. Y. GAO and Z. QIN: A chance constrained programming approach for uncertain p-hub center location problem. Comput. Ind. Eng. 102 (2016), 10-20.

16. M. GalaviI: The inverse 1-median problem on a tree and on a path. Electron. Notes Discret. Math. 36 (2010), 1241-1248.

17. A. J. Goldman: Optimal center location in simple networks. Transportation Sci. 5 (1971), 212-221.

18. X. GuAn and B. Zhang: Inverse 1-median problem on trees under weighted Hamming distance. J Global Optim. 54 (2012), 75-82.

19. X. GUAN and B. ZHANG: Inverse 1-median problem on trees under weighted $l_{\infty}$ norm. LNCS. 6124 (2010), 150-160.

20. S. L. HAKIMI: Optimum locations of switching centers and the absolute centers and medians of a graph. Oper. Res. 12 (1964), 450-459. 
A Hybrid Algorithm for the Uncertain Inverse $p$-Median Location Problem

21. S. L. HAKImI: Optimum distribution of switching centers in a communication network and some related graph theoretic problems. Oper. Res. 13 (1965), 462-475.

22. X. HuAnG and D. HAO: Modelling uncapacitated facility location problem with uncertain customers' positions. J. Intell. Fuzzy Syst. 28 (2015), 2569-2577.

23. J. HATZL: 2-balanced flows and the inverse 1-median problem in the Chebyshev space. Discrete Optim. 9 (2012), 137-148.

24. J. Kennedy and R. C. EBerhart: Particle swarm optimization. ICNN'97. 4 (1995), 1942-1948.

25. B. LiU: Uncertainty theory. 2nd ed., Springer-Verlag, Berlin (2007).

26. B. LIU: Uncertain risk analysis and uncertain reliability analysis. J. Uncertain Syst. 4 (3) (2010), 163-170.

27. X. LIU: Uncertain programming model for location problem of multi-product logistics distribution centers. Appl. Math. Sci. 9 (2015), 6543-6558.

28. R. F. Love: Facilities location: models and methods. Oper. Res. Ser. (1988).

29. I. Mirzapolis Adeh, F. Baroughi and B. Alizadeh: A modified particle swarm optimization algorithm for general inverse ordered $p$-median location problem on network. Facta Universitatis, Ser. Math. Inform. 32 (2017), 447-468.

30. K. T. NGUYEN: Inverse 1-median problem on block graphs with variable vertex weights. j. optimiz. theory appl. 168 (2016), 944-957.

31. K. T. NGUYen and N. T. L. CHI: A model for the inverse 1-median problem on trees under uncertain costs. Opusc. Math. 36 (2016), 513-523.

32. J. PEnG: Risk metrics of loss function for uncertain system. Fuzzy Optim. Decis. Ma. 12 (2013), 53-64.

33. J. Peng, B. Zhang and S. Li: Towards uncertain network optimization. J. Uncertain. Anal. Appl. 3 (2015), Article 4.

34. Z. QIN and Y. GAO: Uncapacitated $p$-hub location problem with fixed costs and uncertain flows. J. Intell. Manuf. 28 (2017), 705-716.

35. R. Rahmaniani, M. Saidi-Mehrabad and A. Ghaderi: An efficient hybrid solution algorithm for the capacitated facility location-allocation problem under uncertainty. JOIE. DOI: 10.22094/joie.2018.538339 (2018).

36. A. R. SEPASIAN and F. RAhBARNiA: $A n O(n \log n)$ algorithm for the inverse 1median problem on trees with variable vertex weights and edge reductions. Optimization. 64 (2015), 595-602.

37. A. Schobel and D. Scholz: The big cube small cube solution method for multidimensional facility location problems. Comput. Oper. Res. 37 (2010), 115-122.

38. H. D. Sherali and C. H. Tuncbilek: A squared Euclidean distance locationallocation problem. NRL. 39 (1992), 447-469.

39. A. TAMIR: $A n O\left(p n^{2}\right)$ algorithm for the $p$-median and related problems on tree graphs. Oper. Res. Lett. 19 (1996), 59-64.

40. K. E. WANG and Q. YANG: Hierarchical facility location for the reverse logistics network design under uncertainty. J. Uncertain Syst. 8 (2014), 255-270.

41. Sh. WAng, J. WAtAda and W. Pedrycz: Value at risk based two-stage fuzzy facility location problems. TII. 5 (2009), 465-482. 
42. M. R. WAGNer, J. BhADURY and S. PENG: Risk management in uncapacitated facility location models with random demands. Comput. Oper. Res. 36 (2009), 10021011.

43. M. WEN, Z. QIN, R. KANG and Y. YANG: The capacitated facility locationallocation problem under uncertain environment. J. Intell. Fuzzy Syst. 29 (2015), 22172226.

44. K. YANG, Y. LIU and G. YANG: Optimizing fuzzy p-hub center problem with generalizedvalue at risk criterion. Appl. Math. Model. 38 (2014), 3987-4005.

45. K. YAnG, Y. LIU and G. YANG: An improved hybrid particle swarm optimization algorithm for fuzzy p-hub center problem. Comput. Ind. Eng. 64 (2013a), 133-142.

46. B. Zhang, J. Peng and S. Li: Covering location problem of emergency service facilities in an uncertain environment. Appl. Math. Model. 51 (2017), 429-447.

Akram Soltanpour

Faculty of Basic Sciences

Department of Applied Mathematics

Sahand University of Technology, Tabriz, Iran

Fahimeh Baroughi

Faculty of Basic Sciences

Department of Applied Mathematics

Sahand University of Technology, Tabriz, Iran

baroughi@sut.ac.ir

(Corresponding author)

Behrooz Alizadeh

Faculty of Basic Sciences

Department of Applied Mathematics

Sahand University of Technology, Tabriz, Iran 